\title{
EL GERUNDIO INDEPENDIENTE
}

\author{
Gemma Herrero \\ (Universidad de Valladolid)
}

\begin{abstract}
RESUMEN
This paper deals with certain constructions in which gerund seems to fuction as a verbal head in a independent sequence, not governed syntactically. First of all, we proceed to a classification of the sequences according to two main factors: grammatical modality and utterance type (contextual and not contextual). Afterwards, we analyze syntactically the G.I. constructions, establishing the gerund function and kind of syntactic unit. We also approach the semantics of the independent gerund and, finally, we make differences between G.I. sequences and other similar constructions.
\end{abstract}

O. El presente trabajo tiene como objeto el estudio de ciertas construcciones en las que el gerundio funciona como núcleo verbal de una secuencia que constituye enunciado. Dicho en otras palabras, el gerundio se actualiza en el discurso como verbo - con las características sintácticas de esta categoría-y constituye una oración independiente, puesto que presenta autonomía sintáctica, ya que no está incluida en una forma mayor (Luna Traill, 1980).

En la limitada bibliografía existente sobre el tema, dicho gerundio recibe la denominación de Gerundio independiente (Vid. D. Magallanes, 1970; C. Bobes, 1975; E. Luna, 1980; N. Donni, 1983; M. J. Albalá,1988; H. Otálora,1992)'.

\footnotetext{
1 Algunas autoras separan el gerundio independiente ( ${ }_{i}$ Siempre cantando $\mathfrak{i}$ ) del gerundio coordinado (Salió a la calle y gritando sin parar). Sin embargo, creemos que ha de ser considerado de igual forma ya que en ambos casos el gerundio es núcleo verbal de su secuencia y no depende de ningún elemento externo. La ley de multiplicidad brinda la posibilidad de unir elementos equifuncionales, en este caso, construcciones con verbo en forma personal y en gerundio, o bien, dos construcciones con verbo en genundio (iTú paseando y yo estudiandoi).
} 
Las secuencias de gerundio independiente son características del estilo hablado y de los registros informales del español; por ello no es de extrañar que su análisis haya surgido al hilo de la investigación sobre los materiales publicados para el estudio del habla de las principales ciudades de Iberoamérica y España.

1. En la casi totalidad de los trabajos citados se proporciona una brevísima descripción del gerundio independiente, sin realizar un análisis pormenorizado del mismo, y, después, se lleva a cabo la clasificación de las oraciones en las que funciona, atendiendo simultáneamente al tipo de estructura sintáctica que presentan y a los valores que posee el gerundio (histórico, narrativo, modal, etc.).

Los resultados son bastante similares. E. Luna (1980: 115) y también H. I. Otálora (1992) distinguen los siguientes tipos de gerundio independiente:

a/ en el contexto pregunta-respuesta :

* —¿Qué están haciendo?

-Grabando.

* -Estuve en S.Cosme dos años.

${ }_{-}$¿Cursando qué?

b/ en sustitución de un verbo conjugado (el llamado gerundio histórico):

* Todos con sus linternitas alumbrándose para...y se juntaban en grupitos $y$ andaban conmigo.

M. J. Albalá (1988: 201) añade a los dos tipos anteriores

c/ el gerundio con valor locativo ${ }^{2}$ : mucho.

* Pero luego la parte esta de pasando ya a Dinamarca para arriba, ee...cambia

D. Magallanes (1970) observa, además, otras variantes:

d/ gerundio como verbo dominante en cláusulas de sentido adversativo, de acusado carácter emocional (p. 239):

* Uno superándose, estudiando carreras y carreras, para que llegue cualquiera a decirle lo mal que vive.

e/ el gerundio modal (subordinado mentalmente a un verbo regente implícito que el contex to deja entrever o imaginar, p. 238):

* Ya traía yo una proparación... pues de formación puramente personal, ¿verdad?: urgando aquí, allá, tratando de entender ${ }^{3}$.

2 No se entiende bien la consideración de este genundio como independiente, puesto que, como ella misma señala, equivale a una preposición o adverbio de significación locativa sin perder por ello su carga verbal. En otras palabras, no posee independencia sintáctica.

3 Según esta autora, «los gerundios están lógica - aunque no gramaticalmente-subordinados 


\section{f/gerundio en frases exclamativas:}

* No, pues me parece muy bien: ¡Tú imponiendo la moda!.

Después de esta breve revisión, puede observarse que las clasificaciones realizadas son bastante heterogéneas; se mezclan parámetros de tipo sintáctico, semántico, pragmático y enunciativo; por otra parte, el análisis gramatical de las secuencias es escaso o inexistente. Apenas se establece la función sintáctica que el gerundio desempeña y, cuando se hace, no se justifica.

En una línea distinta y desde otra perspectiva C. Bobes (1975) se plantea exclusivamente el valor gramatical del gerundio independiente en dos tipos muy precisos de enunciados, sin considerar ningún otro: $1 /$ descriptivos, tales como titulares, explicaciones a pie de foto etc...(Ranas pidiendo rey);

2/ exclamativos, como los ya vistos.

En ambos casos concluye que, como tal, el gerundio independiente gramaticalmente no existe, no constituye núcleo de sentencia u oración.

2. Ante posiciones tan opuestas como las reseñadas parece conveniente realizar un estudio más detallado que tenga en cuenta diversos puntos de vista y considere un número más amplio y representativo de enunciados con gerundio, para poder determinar si se puede hablar del gerundio independiente y en qué términos debe hacerse.

En primer lugar realizaremos la clasificación de los enunciados con gerundio independiente atendiendo a dos factores: (2.1) la modalidad de enunciación (A. Meunier, 1974: 13-18) o modalidad oracional y (2.2) el tipo de enunciado considerado (enunciados contextuales y no contextuales).

2.1. El gerundio independiente admite todos los tipos de modalidad de enunciación (declarativa, exhortativa, interrogativa y exclamativa):

2.1.1.Declarativa: El gerundio se usa preferentemente en secuencias narrativas o descriptivas en las que predomina la localización temporal en el pasado, por lo que algunos autores denominan al gerundio de estas secuencias históriconarrativo:

* Y ese me colocó. Allí aprendí a cortar el pelo, pero acabé encontrándome a disgusto y me marché también. Y dando vueltas hasta hoy, de una parte a otra.

* Y la llamo ¡Lucita!, que se viniese con nosotros, que qué hacía ella sola y no contesta. Nosotros hablándola como si tal cosa y ella ahogándose ya.

a un verbo no expreso: «una preparación que logré urgando..». Resulta forzado admitir una subordinación de tipo lógico, más todavía cuando hay que suponer un posible verbo regente. 
No obstante, en otros casos, la localización temporal corresponde al presente, puesta de manifiesto por el contexto de enunciación o por expresiones temporales:

* Y esos polis siempre metiendo las narices.

* - ¿Qué haces, Bea?

- Pues ya ves. Estudiando para el examen de mañana.

Se incluye también en la modalidad declarativa el llamado gerundio de posterioridad ${ }^{4}$-muy poco usado en general, incluso en situaciones formales- :

* Declara que (..) percibió gritos de socorro provenientes de la parte del río, acudiendo prontamente en compañia de tres de sus compañeros y distinguiendo acto seguido desde la orilla el bulto de una persona.

2.1.2. Interrogativa. El gerundio independiente se utiliza, sobre todo en registros informales, en interrogativas orientadas (exclamativas, confirmativas o hipotéticas) marcadas por la actitud subjetiva del hablante ${ }^{5}$ :

* Qué, ¿Tomando el solete?

* ¿Qué? ¿Pedro vendiendo cerillas en los semáforos?

* ¿Con que metiendo otra vez las narices en mis asuntos, eh?

En algunos enunciados contextuales el valor de la interrogativa es el de pregunta:

* - Pedro ya ha empezado a currar.

- ¿Haciendo qué?.

2.1.3. Exhortativa. En situaciones informales situadas temporalmente en el presente es muy usual el gerundio en oraciones imperativas:

* ¡Andando! El berzas ese nos quiere ver ya.

* Venga, estudiando todos como benditos hasta las diez.

* Circulen, circulen, andando.

* ${ }_{i}$ Fuera, fuera, a correr!. Tú, Petri, dale un besito a tu padre y arreando para fuera.

4 El G.P. expresa una acción temporalmente posterior a la de otro verbo con el que se relaciona paratácticamente, por lo que algunos autores admiten que el gerundio sustituye a un verbo en forma personal (A. Badía, 1964; J. Bouzet, 1953; J. M. Lope Blanch, 1962; E. Luna, 1980). Otros autores lo rechazan. C. Bobes, $1975,-$ que recoge las opiniones a favor y en contra sobre el temaafirma que el gerundio no sustituye a un elemento paratáctico porque indica siempre relaciones de subordinación; por tanto si el gerundio no se relaciona en subordinación con el verbo personal (puesto que no puede indicar una acción posterior que modifique la acción anterior expresada por el verbo principal), no hay por qué usar el gerundio, la expresión adecuada será una coordinación.

5 V. Escandell (1988): la interrogación orientada es la petición no imparcial de respuesta, en la que el emisor concede más probabilidades a una de las respuestas, ya que posee diferentes grados de certeza - mayor o menor - sobre lo que expresa en su enunciado. Algunas están muy próximas a las interrogativas reflejas de Fernández Ramírez (1959). 
E. Lorenzo (1966) señala que este uso del gerundio es propio del registro coloquial, como derivado de otro giro, también informal y utilizado por gente culta que sustituye al imperativo, la forma estar+gerundio: ; Ya te estás callando!. Actualmente tiene mayor vigencia el uso del gerundio aislado con un patrón entonativo apelativo muy acusado, lo que facilita su uso en oraciones de mandato, pero no en las de ruego.

2.1.4. Expresiva. Un rasgo característico del registro coloquial (en todos los niveles socioculturales) es el uso del gerundio independiente en situaciones de marcada expresividad en las que la actitud del hablante se hace explícita:

* ¡Ya, lo que faltaba pal duro! ¡La niña liando canutos!

* ¡Qué pesados! ¡Siempre dando vueltas a lo mismo! ¡Ni de viejos cambian!.

Con notable frecuencia se utiliza el gerundio independiente en enunciados expresivos compuestos por dos secuencias coordinadas que se contrastan fuertemente: horas!

* ¡Muy bonito! ¡Tu padre todo el día trabajando y tú vagueando a todas

* ¡Todo dios esperando que descubra algo y el tío durmiendo!».

En estos casos existe una especial configuración de la línea melódica del enunciado con tonema ascendente detrás del primer miembro.

Se puede afirmar, a la vista de lo expuesto, que el gerundio independiente no presenta ninguna restricción de uso en relación con la modalidad de enunciación y que, contrariamente a los datos expuestos en los trabajos citados, no es el gerundio histórico-narrativo (modalidad declarativa) el más usual, sino los empleados en enunciados interrogativos, expresivos y exhortativos, marcados por la entonación de elipsis o enfática (Skydsgaard, 1977: 1125-1126), que afecta a las entonaciones interrogativa, volitiva y emocional, permitiendo «no sólo subrayar, sino también aislar unidades que en una entonación neutral presupondría otras unidades de oración». De esta forma la entonación enfática o marcada permite considerar al gerundio independiente como núcleo del enunciado, sin necesidad de sobreentender otros elementos, a la vez que hace explícita la actitud del hablante ante el enunciado (sorpresa, asombro, enfado, ironía, etc.). Este hecho explica el notable uso del gerundio independiente en enunciados que admiten la entonación marcada.

2.2. El gerundio independiente se actualiza en enunciados contextuales (o enunciados en contexto, Martinet, 1986) y no contextuales.

2.2.1. Los enunciados contextuales son aquellos enunciados interpretables 
únicamente por su relación con el contexto lingüístico previo. La mayoría corresponden al contex to pregunta/respuesta:

* - ¿Y los pequeños?

-Pues mira, los niños creciendo y avanzando en la vida y nosotros quedándonos atrás.

* - ¿Cómo lo sobrellevas?

-Pues nada, leyendo mucho.

* - ¿Qué haces tan solo por aquí?

-Dando un paseo a ver si me despejo.

También en intervenciones de un mismo interlocutor se encuentran enunciados en gerundio que informativa y significativamente se relacionan con emisiones anteriores:

* Si es lo pesados que se ponían y la manera tan ignorante y tan sin gracia de hablar con una chica. Te sientes como gallina en corral ajeno. ¡Deseando marcharte cuanto antes!.

* ¿Y ese qué hace? ¿Durmiendo todavía?.

* Ahora estoy trabajando bastante.jMenos mal! Haciendo sustituciones y pasando trabajos a máquina!

* ¡Juanito!,¡Bájate de ahí inmediatamente! ¡Y ya estáis volviendo ahora mismo los tres para acá! ¡Pero volando!

En ciertos enunciados contextuales, además del estrecho vínculo semántico, existe una evidente relación sintáctica que se analizará más adelante (cómo/ gerundio; qué tal/gerundio; perífrasis/gerundio).

2.2.2. Enunciados no contextuales. Se incluyen aquí las secuencias de gerundio independiente que pueden ser interpretadas sin la presencia del contex to lingüístico anterior. En este caso hay dos factores que posibilitan en gran medida la presencia del gerundio independiente, la entonación marcada (vid.supra) y la situación en la que se produce el enunciado.

La entonación enfática, como se ha indicado, incide determinantemente en la sintaxis, aislando al gerundio y dotándolo de independencia sintáctica, al tiempo que confiere relevancia al aspecto durativo del gerundio; en consecuencia, desempeña las funciones integradora-delimitadora y expresiva. La mayor parte de enunciados con modalidad expresiva, exhortativa e interrogativa son de este tipo:

* ¡El pesado ese todo el santo día molestando!

* iAhora pasando de explicaciones!

La situación permite la omisión de elementos, innecesarios informativamente, puestos de manifiesto por el entorno comunicativo. De esta forma quedan 
destacados los que se hacen explícitos en el enunciado. No es extraño, pues, que bastantes gerundios independientes puedan ser relacionados, gramaticalmente, con originarias perífrasis constituidas por un verbo conjugado y el gerundio: la situación evidencia los valores temporales y personales (pueden además estar expresados por otras unidades lingüísticas), lo que hace superflua la presencia del verbo en forma personal; el gerundio independiente, por tanto, potencia el valor durativo de la acción que interesa destacar.

En las interrogaciones orientadas el peso de la situación es considerable, sobre todo en las exclamativas, ya que se parte de un estado de absoluta certeza, propiciado en muchos casos por el entorno comunicativo (enunciados en situación). Por ejemplo, alguien está en la cocina haciendo la comida y le decimos:

$$
\text { * Qué, ¿haciendo la comida?; }
$$

o bien en un bar donde alguien juega a las cartas se dice

* ¿Así que echando la partidita, no?

Asimismo los enunciados imperativos, por razones obvias, son situacionales:

* ¡A Ahora todos vosotros escuchando al director con toda atención! ¡Nada de murmullos!

y bastantes de modalidad expresiva: somos testigos de un suceso que nos hace exclamar:

* ¡Si no lo veo no lo creo! ¡Pepe planchándose los pantalones!

En bastantes casos es preciso considerar al mismo tiempo ambos factores, ya que actúan interrelacionados: determinadas circunstancias originan un tipo especial de entonación.

Teniendo en cuenta los factores señalados, se puede afirmar que los enunciados de gerundio independiente son básicamente no contextuales $\mathrm{y}$, con bastante frecuencia, propios de modalidades marcadas (exhortativa, interrogativa y expresiva), debido a la incidencia de la entonación y la situación en el discurso.

3. Tradicionalmente se reconoce la capacidad plurifuncional del gerundio, como adverbio, verbo y en algunos casos como adjetivo (se admite el valor explicativo, pero no el especificativo). También se ha señalado que su función potencial se actualiza en el texto, adquiriendo entonces las propiedades sintácticas de una u otra categoría (E. Luna, 1980: 20).

El gerundio independiente, como se ha indicado, se actualiza en el discurso como verbo central del enunciado. Ahora bien, las diferencias existentes entre unos y otros enunciados hacen conveniente su estudio por separado. 
3.1. Consideraremos en primer lugar los enunciados no contextuales. Revisando las muestras del gerundio independiente en este tipo de enunciados, se puede afirmar que en ellos el gerundio es efectivamente el núcleo verbal de la oración, ya que no depende sintácticamente de ningún elemento externo. Así pues, se trata de oraciones cuyo núcleo verbal no es un verbo en forma personal, sino un gerundio que tiene una relación predicativa con un sujeto ${ }^{6}$. Este aparece manifiesto, si no ha aparecido con anterioridad o no puede ser identificado por la situación o el contexto:

\section{* ${ }_{i}$ Pedro planchándose los pantalones!.}

Muchos de los autores que estudian este tipo de gerundio son de idéntica opinión, si bien consideran que su uso como núcleo verbal de la oración es posible porque el gerundio sustituye a otras formas verbales, habitualmente a formas de pretérito (D. Magallanes, 1970; E. Luna, 1980; N. Donni, 1983; H. Otálora, 1992). Sin embargo C. Bobes (1975: 9) afirma que «no pueden considerarse estos usos como gerundios independientes, en el sentido gramatical de que constituyan núcleos de sentencia u oración, porque responden a una estructura lingüística más amplia (...) de enunciados que se completan con otro sistema de signos no lingüísticos: gráficos, situacionales, etc.

Realmente no se encuentra ningún dato que impida la consideración del gerundio como núcleo verbal de la oración que forma. El hecho de interpretar estas secuencias como enunciados no afecta a la consideración sintáctica que se haga del gerundio.

Es conveniente, por tanto, señalar cómo es posible que el gerundio, habitualmente subordinado a otra unidad, sea el núcleo verbal de una oración y, en consecuencia, pueda aceptarse su independencia. Para ello hay que tener presente que, con gran frecuencia, el gerundio forma parte de perífrasis en las que el verbo principal o auxiliado es el gerundio (en este caso domina el valor verbal y no el adverbial) y el otro verbo un mero auxiliar (generalmente de movimiento o estado, y gramaticalizado en la mayoría de los casos) que manifiesta los morfemas verbales y está inhabilitado para la selección de sujeto y complementos (L. Gómez, 1988: 9-18).

Desde el punto de vista informativo el término más importante de la perífrasis es el gerundio, ya que además del significado, aporta a la acción el aspecto durativo y el valor imperfectivo? ${ }^{7}$. No es extraño, pues, que sea en este constituyente perifrástico en el que se centre la atención del hablante, sobre todo en registros

6 Aunque el gerundio paradigmáticamente es indiferente o neutro respecto al rasgo 'persona', al actualizarse en el discurso puede mantener una relación predicativa con cualquier persona gramatical como sujeto (C. Bobes, 1975: 31; N. Donni, 1983: 205).

7 C. Bobes (1975: 13), aunque considera que en la perífrasis el gerundio mantiene su valor adverbial, afirma también que el gerundio aporta el valor semántico y la forma de acción, relegando 
informales, prescindiendo de la información puramente gramatical que aporta el verbo auxiliar y que, en bastantes casos, es recuperable por el contex to o la situación comunicativa. Por ello, en el español hablado y coloquial, es habitual que el verbo auxiliar, al no ser necesario desde el punto de vista informativo, desaparezca y sea entonces el gerundio el que asuma los valores de la perífrasis, potenciando el carácter durativo y neutralizando otras posibles variantes: incoativa, iterativa, ingresiva ..etc.

En consecuencia el gerundio sigue funcionando como núcleo verbal, de modo semejante a como lo hacía en la originaria perífrasis (aunque en ella los dos términos actuaban conjuntamente como núcleo verbal), pero sin el apoyo del verbo auxiliar.

3.2. En los enunciados contextuales hay que considerar la existencia o no existencia de relación sintáctica entre el enunciado en gerundio y los enunciados previos.

3.2.1. Algunos enunciados, aunque desde el punto de vista semántico e informativo se relacionan con el contexto previo, no guardan con él relaciones de tipo sintáctico:

* - ¿Qué haces?

- Estudiando.

* ¿ ¿Y los pequeños?

- Pues mira, los niños creciendo y avanzando en la vida y nosotros quedándonos atrás.

* _ ¿Y ese qué hace? ¿Durmiẹndo todavía?

Por ello los consideramos de forma idéntica a los enunciados no contextuales: el gerundio, sin necesidad del verbo auxiliar, funciona como núcleo verbal del predicado en una oración que puede llevar o no expreso el sujeto. De esta manera potencia el valor durativo e imperfectivo de la acción verbal.

3.2.2. En otros enunciados, por el contrario, el vínculo sintáctico con el contexto previo es evidente:

* - ¿Cómo lo sobrellevas?

- Pues nada, leyendo mucho.

* ¿QQué tal lo pasasteis en vacaciones?

- Nadando todo el día.

* Ahora estoy trabajando bastante. ¡Menos mal! Haciendo sustituciones y pasando trabajos a máquina.

a un segundo plano, o incluso anulando, el significado del verbo personal. S. Lyer $(1932,12)$ también destaca que en las perífrasis de gerundio y verbos de movimiento, frecuentemente el verbo auxiliar pasa a ser un verbo puramente formal, mientras que el gerundio pasa a un primer plano, destacando la acción que expresa. 
En estos casos, la secuencia constituye enunciado: el contexto, la situación y el patrón entonativo posibilitan que sea reconocida como tal, por tanto se trata de enunciados contextuales, en situación o mínimos (A. Martinet, 1987). La cuestión fundamental es determinar qué tipo de secuencia sintáctica es la que constituye el enunciado y, en consecuencia, qué función desempeña el gerundio en ella:

a/ se trata de una oración en la que se eliden, por su relación con el contexto lingüístico anterior, los elementos conocidos, haciéndose explícitos sólo los nuevos; el gerundio funciona en la oración 1/ como núcleo verbal de una proposición en función de $\mathrm{C}$. Circunstancial $-\mathrm{u}$ otras posibles funciones oracionales-, adyacente del verbo central de la oración, no expreso por ser recuperable al relacionarlo con el contex to previo ((lo sobrellevo) leyendo mucho); $2 /$ como núcleo verbal de una perífrasis, conjuntamente con el verbo auxiliar no expreso (Estoy trabajando bastante. ¡ Menos mali (Estoy) haciendo sustituciones y pasando a máquina). Lógicamente si se acepta esta propuesta no se podría seguir considerando al gerundio como independiente.

b/ se trata de fragmentos (secuencias elípticas contextualmente, de carácter no oracional. vid. M. L. Hernanz, 1987: 110) no analizables desde el punto de vista sintáctico. El gerundio funciona como núcleo de la secuencia, por lo que es independiente.

4. En la mayor parte de los estudios citados se destaca el carácter durativo como el valor semántico más importante del gerundio. Puesto que la forma de gerundio que se actualiza es siempre la simple, hay que sumar al valor durativo el aspecto imperfectivo. Evidentemente este es el significado habitual del gerundio independiente, la «indicación, desde el punto de vista aspectual, de una acción no terminativa, esto es, en su desarrollo» (N. Donni de Mirande, 1983: 205); incluso la modalidad durativa que manifiesta el gerundio neutraliza otras posibles variantes de la forma de la acción verbal: incoativa, iterativa, ingresiva, etc. La acción en desarrollo, o valor durativo del gerundio, adquiere en algunos casos una relevancia especial, acentuada por la inclusión de expresiones temporales con significado de acción continua, habitual o repetida (Toda la vida, todo el día, siempre... etc.):

* ¡Qué pesado! ¡Siempre dando vueltas al mismo tema!

* iA su edad y todavía creyendo en los Reyes Magos!

* Es un clima muy triste. Todo el santo día lloviendo!

Algunos autores, si bien indican que el uso del gerundio independiente proporciona a la descripción límites temporales imprecisos, destacan su uso para expresar acciones durativas situadas en el pasado (Gerundio históriconarrativo), «como equivalente no de un pretérito de indicativo, sino de un 
imperfecto, de acuerdo con el valor aspectual básicamente imperfectivo del gerundio»; en otros casos el G.I. no alterna con formas verbales del pretérito sino que está en sustitución de ellas o, incluso, alterna con expresiones de carácter nominal (D. Magallanes, 1970: 236; M. J. Albalá, 1988).

Respecto a estas afirmaciones hay que hacer notar que el gerundio es una forma paradigmáticamente no marcada, neutra en cuanto al tiempo y la persona (J. Bouzet, 1953; M. Molho, 1971; E. Alarcos, 1978), por tanto no puede indicar él mismo la perspectiva temporal en la que se sitúa la acción verbal; esto no impide que el gerundio independiente, aunque no manifieste el tiempo, se vea afectado en el discurso por la orientación temporal del enunciado, puesta de manifiesto por expresiones temporales, por el contex to linguístico previo o por la situación comunicativa en la que se actualiza el enunciado. Esto explica que en ocasiones el valor durativo e imperfectivo del gerundio quede determinado por la acción perfectiva del enunciado; así el valor durativo se da por acabado en un momento determinado:

* Y ese me colocó. Allí aprendí a cortar el pelo, pero acabé encontrándome a disgusto y me marché también. Y dando vueltas hasta hoy, de una parte a otra.

En conclusión, el gerundio independiente manifiesta en el discurso un valor durativo e imperfectivo y, aunque es una forma neutra en cuanto al tiempo, asume, justamente por ello, la orientación temporal de todo el enunciado.

5. Por último se hace necesario distinguir los enunciados de gerundio independiente de otras secuencias con gerundio muy semejantes a ellos, al menos aparentemente.

5.1. En determinadas construcciones el gerundio parece ser independiente, puesto que no está subordinado al núcleo verbal de la oración, por lo que manifiesta cierta autonomía respecto al verbo central:

\footnotetext{
* Hablando de otra cosa, ¿Por qué no has ido esta mañana a clase?

* Cambiando de tema, Juan viene esta tarde. Vete a buscarle, si no te importa.

* Hablando de Pedro, ¿Qué sabes de él?
}

Se trata de elementos textuales, relacionados con el acto de enunciación del mensaje, que sirven para introducir el tema del enunciado (son, en estos casos el tópico del enunciado) o indicar los cambios que se producen en el mensaje. No dependen sintácticamente del verbo central de la oración, pero, en tanto que elementos marcadores de enunciación, modifican a todo el enunciado que contiene al verbo central de la oración, puesto que en gran medida justifican la modalidad de enunciación de las secuencias que siguen a continuación (Hablando 
de otra cosa, pregunto $X$, declaro $X$, etc). Por ello, puede determinarse que estas construcciones desempeñan la función de modificador clausal ${ }^{8}$.

5.2. En secuencias coordinadas es frecuente el uso del gerundio en el segundo miembro de la construcción, coordinado copulativa o adversativamente a verbos en forma personal:

* Dicho esto me voy, y pitando.

* Baja a la bogeda a por el vino, pero corriendo.

* Por ahí también se llega, pero torciendo a la derecha.

No se pueden considerar estos gerundios como independientes, ya que dependen sintácticamente de un verbo en forma personal, elidido en el segundo miembro de la construcción coordinada'. El gerundio funciona como adyacente del núcleo verbal, como adverbio modal que modifica al verbo elidido (me voy, pero me voy pitando), tanto en los casos en los que aparece aislado, como en los que lleva elementos oracionales - como núcleo verbal de una proposición adverbial-.

5.3. En titulares periodísticos y publicitarios, explicaciones a pie de fotografías o ilustraciones pueden aparecer enunciados nominales en los que aparece un gerundio que, en un primer momento, pudiera ser considerado como independiente, por no estar relacionado con ningún verbo:

* Manifestantes abucheando a las autoridades municipales.

* Niños jugando en el nuevo parque.

* Bomberos auxiliando a los vecinos del inmueble siniestrado.

Como ya señaló C. Bobes (1975: 8-9), no puede considerarse que en estos casos el gerundio sea núcleo verbal de la oración, debido a que el verdadero núcleo es el elemento nominal que aparece en primer lugar (manifestantes, niños, bomberos), modificado por un gerundio de carácter adjetivo que actúa como adyacente suyo ${ }^{10}$. Esto no impide que el gerundio pueda llevar elementos oracionales propios.

8 G. Rojo y T. Jiménez Juliá (1989: 142) reconocen que la claúsula presenta la posibilidad de poseer un modificador general que actúe sobre el resto de la claúsula (el núcleo) al que denominan modificador clausal.

$9 \quad$ La elipsis gramatical del verbo es posible en las construcciones coordinadas, puesto que se cumplen las condiciones estructurales necesarias para tal elisión: se realiza fónicamente alguna forma que constituye el predicado de la oración (el gerundio en este caso) y existe un antecedente (el verbo expreso en el primer miembro de la construcción coordinada) que permite interpretar el verbo elidido de forma adecuada (Vid. M. L. Hernanz y J. M. Brucart, 1987: 133).

10 C. Bobes (1975) ha explicado y justificado el uso adjetivo del gerundio, rechazado por la mayoría de los gramáticos. En primer lugar, hay que tener presente la ausencia en el sistema verbal español de un adjetivo verbal activo y presente, valor que asume el gerundio en los contextos en que funciona como adyacente nominal. Además, debido al paralelismo funcional del adverbio respecto al núcleo verbal y del adjetivo respecto al núcleo nominal, se puede admitir la transposición funcional del gerundio a adjetivo, hecho permitido por el sistema, aunque rechazado por la norma. 


\section{REFERENCIAS BIBLIOGRÁFICAS:}

ALARCOS, E. (1978): Estudios de gramática funcional del español, Madrid, Gredos.

ALBALA, M. J. (1988): Contribución al estudio del gerundio en la lengua española hablada de Madrid, Madrid, Universidad Complutense.

BADIA MARGARIT, A. Mª (1964): «El gerundio de posterioridad», en Presente y futuro de la lengua española, I, Madrid, OFINES, pp. 287-295.

BOBES NAVES, C. (1975): «Sistema, norma y uso del gerundio castellano», RSEL, 5.1, pp. 1-34.

BOUZET, J. (1953): «Le gérondif espagnol dit de postériorité», Bulletin Hispanique, 55, pp. 349-374.

DÍAZ BAUTISTA, M. C. (1986): Aspectos sintácticos y semánticos del gerundio en español, Madrid, Imprenta Saba.

DONNI DE MIRANDE, N. E. (1983): «Sobre el uso del gerundio en Rosario (Argentina), Philologica Hispaniensia in honorem M. Alvar, II, pp. 191-207. ESCANDELL, V. (1988): La interrogación en español: semántica y pragmática, Madrid, Universidad Complutense.

FERNÁNDEZ, S. (1959): «Oraciones interrogativas españolas», BRAE, 39, pp. 243-276.

FERNÁNDEZ, S. (1960):«Algo sobre la fórmula estar + gerundio», Studia Philologica. Homenaje a D. Alonso, II, pp. 509-516.

GÓMEZ TORREGO, L. (1988): Perífrasis verbales, Madrid, Arco-Libros.

HERNANZ, M. L. y J. M. BRUCART (1987): La sintaxis, Barcelona, Editorial Crítica.

LOPE BLANCH, J. M. (1962): «Sobre la oración gramatical», NRFH, XVI, pp. 416-422.

LORENZO, E. (1966): «La expresión de ruego y mandato en español», en $E l$ español de hoy, lengua en ebullición, Madrid, Gredos, pp. 84-96.

LUNA TRAILL, E. (1980): Sintaxis de los verboides en el habla culta de la ciudad de México, México, UNAM.

LYER, S. (1932): «La sintaxe du gérondif dans le Poeme del Cid», RFE, 19, pp. $1-46$.

MAGALLANES, M. D. (1970): «Oraciones independientes de gerundio en el español de México», Anuario de Letras, VIII, pp. 235-239.

MARTINET, A. (1987): Sintaxis general, Madrid, Gredos.

MEUNIER, A. (1974): «Modalités et communication», Langue Française, 21, pp.8-25.

MOLHO, M. (1971): Sistemática del verbo español, Madrid, Gredos.

OTALORA DE FERNÁNDEZ, H. I. (1992): Uso del gerundio en algunas muestras 
del habla bogotana, Santafé de Bogotá, Instituto Caro y Cuervo.

ROJO, G. y T. JIMÉNEZ JULIA (1989): Fundamentos del análisis sintáctico funcional, Santiago de Compostela, Universidad.

SKYDSGAARD, S. (1977): La combinatoria sintáctica del infinitivo en español, II, Madrid, Castalia. 